\title{
Laurence Podselver, Retour au judaïsme? Les Loubavitch en France
}

Paris, Odile Jacob, 2010, 338 p.

Jacques Gutwirth

\section{OpenEdition}

\section{Journals}

Édition électronique

URL : http://journals.openedition.org/assr/23592

DOI : $10.4000 /$ assr.23592

ISSN : $1777-5825$

Éditeur

Éditions de l'EHESS

Édition imprimée

Date de publication : 31 décembre 2011

Pagination : 259

ISBN : 9782713223273

ISSN : 0335-5985

\section{Référence électronique}

Jacques Gutwirth, «Laurence Podselver, Retour au judaïsme? Les Loubavitch en France », Archives de sciences sociales des religions [En ligne], 156 | octobre-décembre 2011, document 156-93, mis en ligne le 26 mars 2012, consulté le 21 septembre 2020. URL : http://journals.openedition.org/assr/23592

DOI : https://doi.org/10.4000/assr.23592

Ce document a été généré automatiquement le 21 septembre 2020

(C) Archives de sciences sociales des religions 


\section{Laurence Podselver, Retour au judaïsme ? Les Loubavitch en France}

Paris, Odile Jacob, 2010, 338 p.

Jacques Gutwirth

\section{RÉFÉRENCE}

Laurence PODSELVER, Retour au judaïsme ? Les Loubavitch en France, Paris, Odile Jacob, $2010,338 \mathrm{p}$.

1 Laurence Podselver a consacré depuis plus de vingt-cinq ans son activité de recherche à l'étude des hassidim de Loubavitch à Paris. Elle avait publié plusieurs articles de qualité sur le sujet et voici enfin un ouvrage très fouillé qui va au-delà de ses travaux antérieurs. Le livre est largement consacré à la présentation des baaléi tshouve, les " maîtres du repentir », c'est-à-dire des juifs revenus à la religiosité judaïque, ce qui est le cas de la plupart des adeptes Loubavitch en France; il s'agit d'un phénomène singulier au sein de ce hassidisme et du mouvement hassidique dans son ensemble.

2 L'ouvrage comporte aussi nombre d'informations qui concernent l'histoire du mouvement Loubavitch, également dénommé Habad, ainsi que l'activité à son siège mondial à Crown Heights, Brooklyn. De plus, on y saisit fort bien la vénération intense des fidèles de France envers le leader charismatique du mouvement, rabbi Menachem Mendel Schneerson (1902-1994), et tout ce que représente pour eux un séjour dans le voisinage du « rabbi », comme on le nomme respectueusement.

3 De nombreuses pages sont donc consacrées aux baaléi-tshouvé. Une grande partie des « retours au judaïsme » ont provoqué des ruptures plus ou moins durables avec les familles; d'abord, ils se produisaient le plus souvent au moment de l'adolescence, avec acquisition brutale d'un nouveau mode de vie et négation de la culture juive nordafricaine du milieu d'origine. D'où, évidemment, l'importance du nouveau groupe d'accueil, comme substitut à la parentèle. Divers récits de « retour », essentiellement féminins, sont présentés en détail. Comme en témoignent ceux-ci, beaucoup de 
Loubavitch sont issus de milieux populaires, aux conditions de vie précaires. Il y a aussi, notamment avec l'émigration en France, déstructuration de la famille étendue et même éclatement du noyau conjugal des parents. L'auteure consacre aussi un chapitre détaillé aux convertis, les non-juifs qui deviennent hassidim de Loubavitch. Elle montre que ces prosélytes affirment "avoir toujours été juifs sans le savoir jusqu'au dévoilement de leur âme (p.136).» La plupart sont originaires de familles catholiques sécularisées. À noter que la conversion officielle n'est pas le fait du mouvement mais bien du consistoire, car c'est un tribunal rabbinique consistorial, beth din, qui l'accorde ou la refuse; or Loubavitch ne dispose pas d'un tel tribunal. L. Podselver observe que si le converti doit démontrer ses connaissances de la Torah, des pratiques et de l'éthique propres au judaïsme, le degré d'intégration de l'impétrant dans le milieu social et ses relations avec les autres juifs sont également des critères pris en compte. Pour parfaire la conversion, il reste pour les hommes la circoncision à l'hôpital par un médecin religieux et pour les femmes l'immersion dans le bain rituel, mikve. L'auteure observe que « l'évaluation du nombre des prosélytes est d'autant plus difficile à obtenir que leur statut au sein du groupe HaBad, n'est pas particulièrement valorisé. Il est d'ailleurs très significatif que je n'ai pu rencontrer que des personnes en voie de conversion et proches de l'étape finale... (p. 137).»

4 Lag ba Omer, jour de réjouissance, au cours de la période de l'Omer, entre la Pâque et Shavouot, fête de la réception de la Torah par les juifs, assure dans la culture Loubavitch la convergence des pèlerinages sur les tombes des saints, traditionnels chez les juifs d'Afrique du nord, et le même culte chez les hassidim. À Paris il n'y a pas de tombe de saint, mais l'auteure décrit les festivités place de la République, où le portrait de Schneerson - substitut de la tombe ? - domine la scène qui a été montée sur place.

Un intéressant chapitre est consacré aux écoles Loubavitch qui scolarisent $22 \%$ des enfants dans une école juive (chiffres de 2006). Il y avait déjà, en 2002, 28400 enfants dans l'ensemble de ces écoles, ce qui représentait une augmentation de $78 \%$ en dix ans ! L. Podselver note que cette hausse est à comparer à un mouvement général vers l'école privée, notamment catholique. Elle remarque également que désormais les enfants vont vivre un cocktail linguistique complexe, avec le français, l'hébreu, le yiddish... et aussi l'arabe à domicile. L'auteure décrit bien la cérémonie de la coupe des cheveux du petit garçon à l'âge de trois ans, lorsqu'il entre dans le monde masculin.

6 Un important chapitre est consacré aux «femmes au cœur du paradoxe de la modernité ». Comme on le sait, selon la Genèse, la femme est un sous-produit de la création, issue de l'homme. Il semble, selon l'auteure, que les femmes sont tiraillées entre la tradition, qui les cantonne essentiellement dans un rôle de mère de famille, et d'autre part la dynamique de la vie moderne, avec notamment les nécessités pécuniaires qui les poussent à travailler. Néanmoins elles trouveraient dans le messianisme du mouvement et les efforts pour l'accomplissement de la fin des temps, la justification de leur rôle ambivalent. On me permettra d'être sceptique quant au rôle de cette idéologie messianique dans la vie quotidienne. Cela dit, le chapitre fourmille d'observations intéressantes concernant les femmes, avec par exemple la description d'une soirée musicale féminine au Palais des congrès, phénomène certes inimaginable au sein d'autres appartenances hassidiques. Quelques pages consacrées à l'apparence féminine, soulignent encore une fois la combinaison étonnante des règles de la tradition et des tributs à la coquetterie. À noter que la plupart des femmes mariées 
portent des perruques alors que traditionnellement le foulard sur la chevelure coupée était prôné par les rabbins orthodoxes.

7 Un chapitre consacré au travail montre, avec un tableau parlant à l'appui, que les fidèles masculins les plus engagés exercent des professions administratives et pédagogiques dans les institutions Habad. Néanmoins nombre de baaléi-tshouve, ont acquis avant leur «retour» des connaissances qui leur permettent de professer des activités modernes - cadres d'entreprise, informaticiens, médecins, dentistes. Bien sûr il y a aussi des adeptes qui pratiquent des professions traditionnelles, associées à la stricte observance religieuse, notamment dans le secteur de la boucherie, de l'épicerie, de la boulangerie.

8 Je regrette que la présentation systématique de l'activité et de l'organisation Loubavitch en France, qui aurait été bien précieuse, apparaisse seulement en filigrane. Si le livre, notamment au chapitre 3, «de Belleville à New York. Voir le rabbi », traite largement du rapport des fidèles au leader charismatique du mouvement, il faut tout de même observer que celui-ci est décédé depuis 1994, il y a donc déjà dix-sept ans. Or, les répercussions de la disparition du rabbi ne sont présentées que dans cinq pages in fine. Comment font donc désormais les « orphelins » du rabbi, qui n'a pas eu de successeur, alors qu'habituellement il y a transmission " dynastique » au sein du hassidisme, celui de Loubavitch inclus? D'autre part, le messianisme extrême de la dernière période de la vie du rabbi, a suscité, dès avant son décès, des expectatives millénaristes intenses au sein du mouvement Loubavitch, et après sa mort, il y a eu croyance chez nombre de fidèles à sa résurrection plus ou moins proche comme messie (sic), ce qui n'est pas allé sans de graves conflits entre ce type de croyants et d'autres refusant cette éventualité, à l'aspect bizarrement christianisant. Or de cette situation qui a dû aussi concerner les fidèles en France, il transparaît fort peu.

Il s'agit en tout cas d'un ouvrage fourmillant d'informations, d'observations et d'analyses précieuses, qui témoigne d'un beau et durable travail d'ethnologue. 